Facilities for the Performance of Fano

Effect Measurements as a Probe of Electron Correlation

J. G. Tobin, S. W. Yu, T. Komesu, B. W. Chung, S. A. Morton, G. D. Waddill

November 14, 2006

Fall 2006 MRS Meeting Boston, MA, United States November 26, 2006 through November 30, 2006 
This document was prepared as an account of work sponsored by an agency of the United States Government. Neither the United States Government nor the University of California nor any of their employees, makes any warranty, express or implied, or assumes any legal liability or responsibility for the accuracy, completeness, or usefulness of any information, apparatus, product, or process disclosed, or represents that its use would not infringe privately owned rights. Reference herein to any specific commercial product, process, or service by trade name, trademark, manufacturer, or otherwise, does not necessarily constitute or imply its endorsement, recommendation, or favoring by the United States Government or the University of California. The views and opinions of authors expressed herein do not necessarily state or reflect those of the United States Government or the University of California, and shall not be used for advertising or product endorsement purposes. 


\title{
Facilities for the Performance of Fano Effect Measurements as a Probe of Electron Correlation
}

\author{
J.G. Tobin ${ }^{1, *}$, S.W. Yu ${ }^{1}$, T. Komesu ${ }^{2}$, B.W. Chung ${ }^{1}$, \\ S.A. Morton ${ }^{1,2, \#}$ and G.D. Waddill ${ }^{2}$ \\ ${ }^{1}$ Lawrence Livermore National Laboratory, Livermore, CA, USA \\ ${ }^{2}$ University of Missouri- Rolla, Rolla, MO, USA
}

\begin{abstract}
Fano Effect measurements are the key to direct observation of the Kondo or spin shielding intrinsic to models of electron correlation. The Fano Effect is the observation of spin polarized photoelectron emission from NONMAGNETIC materials, under chirally selective excitation, such as circularly polarized photons. Below are described three spectrometers, with which Fano Effects measurements have been made.
\end{abstract}

\section{Introduction}

The key measurements are based upon spin-resolving and photon-dichroic photoelectron spectroscopy. True spin-resolution is achieved by the use of a Mini-Mott detection scheme. The photon-dichroic measurements include the variants $\mathrm{x}$-ray magnetic circular and linear dichroism angular distributions (XMCDAD and XMLDAD). Both a multichannel, energy dispersive collection scheme as well as the spin-detecting Mini-Mott apparatus are used in data collection.

\section{Device 1. Spin Spectrometer}

The "Spin Spectrometer" was previously based at the Spectromicroscopy Facility (Beamline 7) at the Advanced Light Source at Lawrence Berkley National Laboratory (LBNL, Berkeley, CA, USA) and is now located at Beamline 4 EPU (Elliptically Polarized Undulator) at the Advanced Photon Source at Argonne National Laboratory near Chicago, IL, USA. The high angular and energy resolution with high throughput is achieved via the use of an 11-inch mean diameter hemispherical analyzer supplied by Physical Electronics. Included in this package is an electron collection lens stack with an adjustable aperture, permitting selection of various angular and sample spot sizes. The novel aspect of our PHI analyzer is that the multi-channel detector has a hole in the center, permitting the direct passage of energy analyzed electrons into the electron optics, without resorting to an electron switchyard. The presence of the hole does cause some problems when the multi-channel (non-spin) detection is being used: an increase in dark and background counts. Dark counts are defined as non-zero electron counting that occurs when the multi-channel detection is "on" but no excitation is striking the sample. Background counts are the counts underlying the elastic photoelectron peaks, e.g., a core- 
level, when actual collection is underway. Regardless, under many conditions, these problems are inconsequential. Spin resolution is achieved by directing the electrons through the optics and into the Mini-Mott detector. (Figure 1) In this case, the high voltages on the channel plates are turned off and the channel plates and anode assembly become part of the first lens stack, directing the electrons into the $90^{\circ}$ spherical sector. The $90^{\circ}$ sector is run at a relatively high pass energy: energy resolution is provided solely by the hemisphere and the photon monochromator. Because the multi-channel detection is at the exit plane of the hemisphere, the imaging of the hemisphere entrance slit onto the multi-channel analyzer is unperturbed and high resolution non-spin counting is achieved. Furthermore, since the spin resolving detection does not require precise imaging, the burden of high-resolution spatial imaging is lifted from the $90^{\circ}$ sectors. This allows the $90^{\circ}$ sectors to be run at high pass energies, optimizing throughput. The $90^{\circ}$ sector serves one salient purpose: it allows the simultaneous resolution of both the vertical spin (x) and the spin along the electron emission direction(-z). These two components are of particular importance in our experiments. After the $90^{\circ}$ sector, the electrons travel through another lens stack, into the Mini-Mott detector. In the Mini-Mott, the electrons are accelerated to $24 \mathrm{kV}$, with four channeltrons positioned horizontally and vertically used for electron counting. While suffering from a relatively low figure of merit, the Mini-Mott has two key advantages to our design. First, it provides essentially turn-key operation with the requirement of no special preparation. Second, it has been shown that the electron optical matching of a spin detector to a large hemisphere can be optimized by the use of a Mini-Mott.

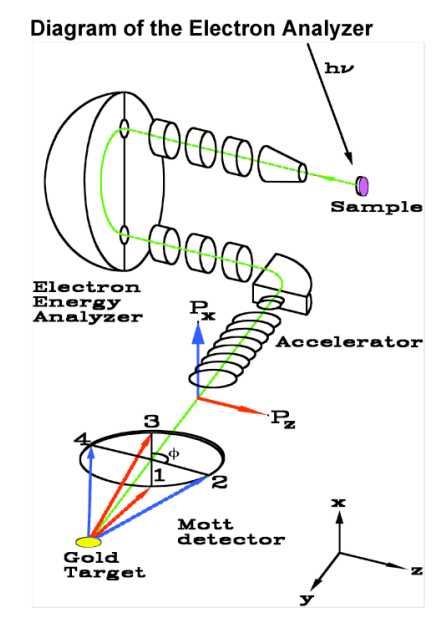

Figure 1a

Diagram of the electron energy analyzer, including the 90 degree sector and MiniMott detector.

\section{Spin Spectrometer}

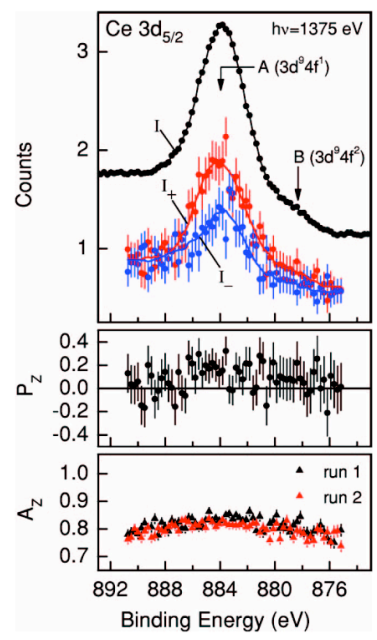

Figure 1b

Shown here is an example of the Fano Effect in a core level of "nonmagnetic" Ce. Circularly polarized x-rays at 1375 $\mathrm{eV}$ were used for the excitation. See Ref. 1 for more details. 


\section{Device 2. UMR Spin Resolving Spectrometer}

A new compact angle resolving spin spectrometer for conducting double polarization experiments has recently been developed at the Advanced Light Source at Lawrence Berkeley National Laboratory(Berkeley, CA, USA). This spectrometer combines a large (11 inch) diameter fixed hemispherical analyzer with a novel rotatable input lens system allowing data with +-1 degree angular resolution to be acquired for any combination of incident and emission angles, including normal incidence / normal emission (Figure 2). The analyzer is equipped with both multichannel detection for high resolution $(50 \mathrm{meV})$ spin integrated spectroscopies, such as XPS and magnetic linear or circular dichroism, and a Mott detector capable of resolving the photoelectron spin polarization simultaneously along the two perpendicular axes of the rotational plane. Rapid switching between spin integrated and spin resolved modes is achieved by focusing the photoelectrons through a small hole in the detector of the hemispherical analyzer and into a compact mini-Mott detector situated immediately behind the channelplates.

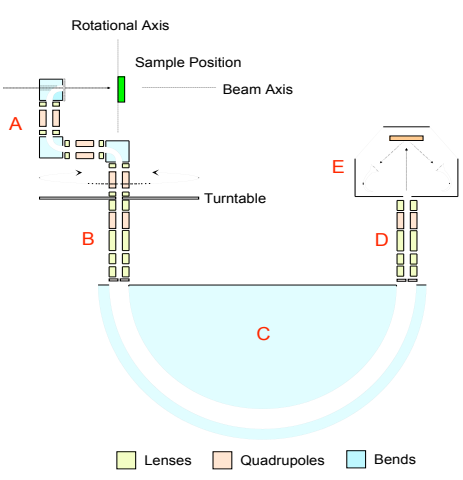

Figure 2a

Shown here is a schematic of the analyzer and lens assembly.

A. Rotating entrance lens assembly

B. Transfer lenses

C. Fixed Large diameter hemispherical analyzer

D. Transfer lenses

E.Fixed spin resolving Mott detector

\section{UMR Spectrometer}

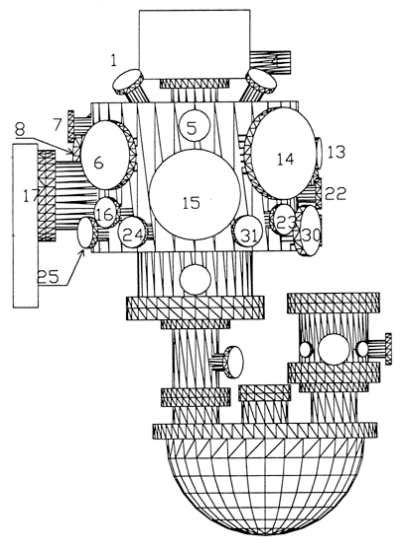

Figure 2b

Schematic of the analyzer system mounted beneath the analysis chamber. The resulting instrumental footprint is smaller than most conventional XPS systems.

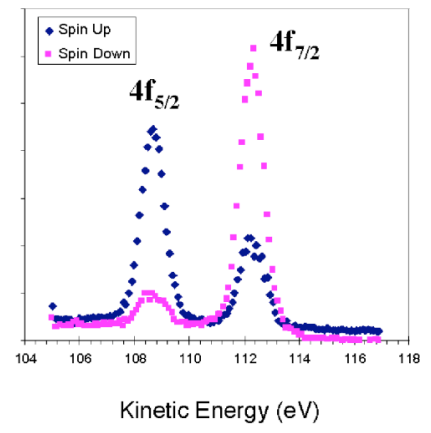

Figure 2c

Fano Effect or "Double Polarization" spectrum of $\mathrm{Au}(111) 4 \mathrm{f}$ peak. For more details, please see Ref 2. 


\section{LLNL Actinide Spectrometer}

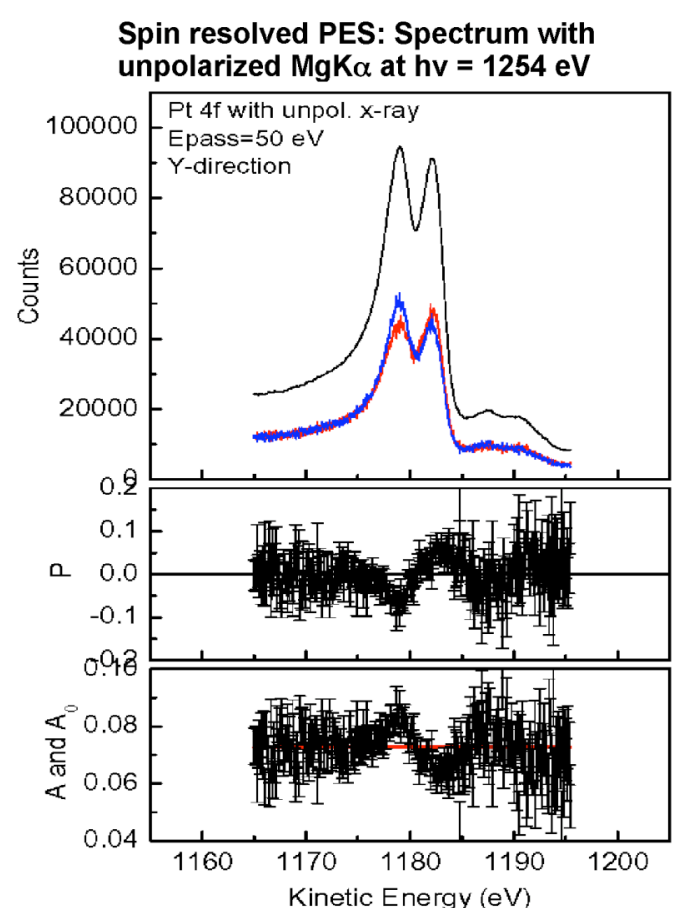

Spin-resolved PES: Diagram of Electron Analyzer from Specs

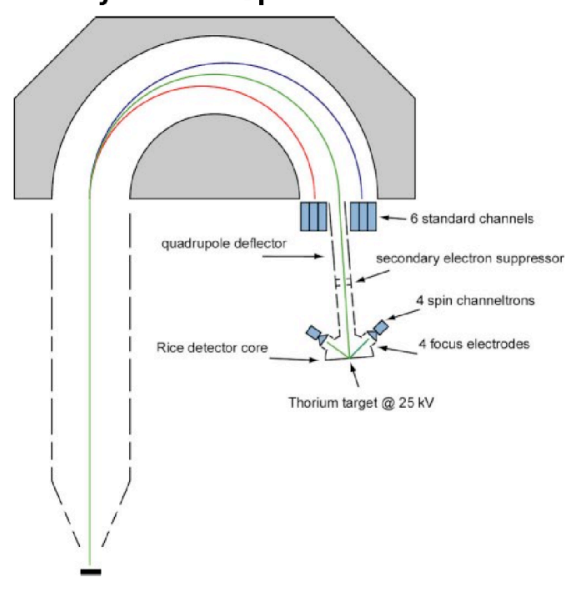

\section{Spin-res PES:MiniMott from Specs}
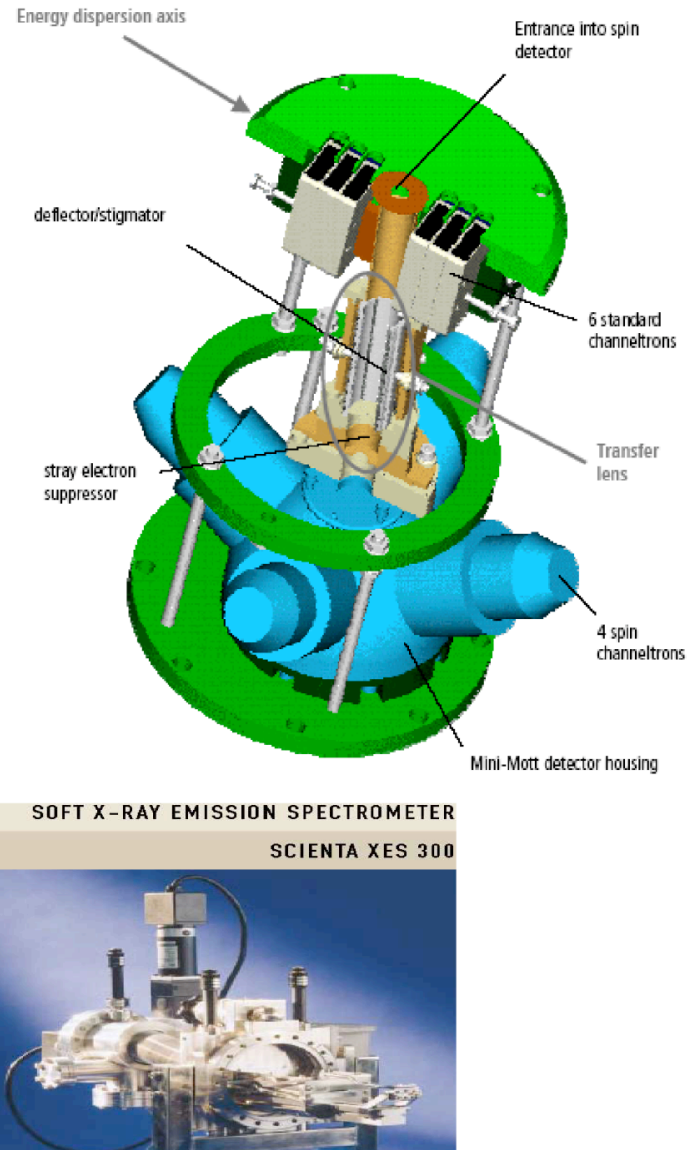

Bremstrahlung Isochromat

Spectroscopy: XES-350

Above is a picture of the XES-350 soft x-ray monochromator from Gammadat Scienta.

Figure 3

Clockwise from the lower left corner: a diagram of the Specs electron energy analyzer and Mini-Mott; a Fano Effect measurement made with the analyzer and a MgK-alpha source $(\mathrm{hv}=1254 \mathrm{eV})$; illustration of the Specs MiniMott; a picture of the XES-350 photon monochromator, which is the detector in tha BIS experiments. 
This combination of fixed hemispherical analyser and in-situ rotating lens assembly results in a number of key advantages over possible alternative spectrometer designs. While the input lens assembly is able to collect photoelectrons from any emission angle the measurement axes of the Mott detector remain fixed along, and perpendicular to, the beam axis. This considerably simplifies the analytical interpretation of angular spin polarization data. Furthermore the complete elimination of the large differentially pumped rotary feedthroughs that are normally required for such large diameter rotating analyzer systems results in a much more compact spectrometer design. Indeed, the positioning of the analyzer beneath the chamber results in a spectrometer footprint that is smaller than most conventional XPS systems (Figure 2). In addition, the small, in-situ lens assembly causes minimal obstruction of the area around the sample without compromising the overall resolution; as would be the case with a conventional small, ARPES style, in-situ rotating hemispherical analyzer. The addition of a small hole through the first bending element also allows for normal incidence / normal emission experiments to be conducted; a high symmetry geometry that is important for verification of certain theoretical models.

\section{Device 3. LLNL Spin Resolving Actinide Spectrometer}

We have built a dedicated spectrometer for performing spin-resolved photoelectron spectroscopy of highly radioactive materials. This spectrometer has the same PES capabilities as the Spin and UMR Spectrometers: Multichannel detection for spinintegrated measurements and a MiniMott for spin-resolved. It also has a capability for BIS with the XES-350 photon monchromator. The spectrometer will include specialized capabilities for handling Pu samples. For example, the long vertical manipulator will allow the isolation of the radioactive byproducts of sample preparation and cleaning from the analysis station yet also permit rapid access of the analysis position, so as to minimize surface corruption after cleaning. (See Figure 3.)

\section{Acknowledgements}

This work was performed under the auspices of the U.S. Department of Energy by University of California Lawrence Livermore National Laboratory under contract No. W-7405-Eng-48.

\section{References}

1. S.W. Yu, T. Komesu, B.W. Chung, G.D. Waddill, S.A. Morton, and J.G. Tobin, Phys. Rev. B 73, 075116 (2006).

2. J.G. Tobin, S.A. Morton, B.W. Chung, S.W. Yu and G.D. Waddill, Physica B 378-380, 925 (2006). 